\title{
Capture of cashew allergen by skin dendritic cells is enhanced in cashew-sensitized mice following epicutaneous delivery
}

\author{
Pierre-Louis Hervé ${ }^{1}$, Benjamin Pelletier ${ }^{1}$, Noémie Assoun ${ }^{1}$, Nathalie Oreal $^{1}$, Audrey \\ Perrin $^{1}$, Jean-Louis Labernardière ${ }^{1}$, and Hugh Sampson ${ }^{1}$ \\ ${ }^{1}$ DBV Technologies
}

October 14,2020

Capture of cashew allergen by skin dendritic cells is enhanced in cashew-sensitized mice following epicutaneous delivery

Running title: Sensitization to cashew leads to increased epicutaneous delivery of cashew allergen

Pierre-Louis Hervé ${ }^{1 *}$, Benjamin Pelletier ${ }^{1}$, Noémie Assoun ${ }^{1}$, Nathalie Oreal ${ }^{1}$, Audrey Perrin ${ }^{1}$, Jean-Louis Labernardière $^{1}$, Hugh A Sampson ${ }^{2}$

* Correspondence should be addressed to P-L.H. (pierre-louis.herve@dbv-technologies.com; +331848611 30)

${ }^{1}$ DBV Technologies, 177-181 avenue Pierre Brossolette, 92120 Montrouge, France

2 DBV Technologies, 12 East 49th Street Tower 49, Suite 4001, New York, NY 10017, USA

Main text word count: 595

\section{Acknowledgments}

Authors acknowledge Isabelle Rombeau for her continuous support in the animal facility. Authors also thank Katharine Bee for proofreading of the manuscript.

\section{Conflicts of Interest}

Dr. Hervé reports personal fees from DBV Technologies, during the conduct of the study; personal fees from DBV Technologies, outside the submitted work.

Benjamin Pelletier reports personal fees from DBV Technologies, during the conduct of the study; personal fees from DBV Technologies, outside the submitted work.

Noémie Assoun reports personal fees from DBV Technologies, during the conduct of the study.

Nathalie Oreal reports personal fees from DBV Technologies, during the conduct of the study.

Audrey Perrin reports personal fees from DBV Technologies, during the conduct of the study.

Jean-Louis Labernardière reports personal fees from DBV Technologies, during the conduct of the study; personal fees from DBV Technologies, outside the submitted work.

Dr. Sampson reports personal fees from DBV Technologies, during the conduct of the study; personal fees from DBV Technologies, outside the submitted work.

To the editor, 
The prevalence of cashew allergy has been rising in industrial countries. ${ }^{1}$ More critically, accidental contact with cashew-containing food is frequently associated with highly severe anaphylactic reactions in allergic individuals. ${ }^{2}$ These elements highlight the need for a safe treatment able to minimize the impact of this life-threatening allergy.

In a recent study published in Allergy, our group evaluated the relevance of investigational epicutaneous immunotherapy (EPIT) to protect against anaphylaxis in a mouse model of cashew allergy. ${ }^{3}$ We demonstrated that epicutaneous patches containing cashew allergens were able to modulate cashew-specific antibody responses and decrease cashew-specific Th2 responses in cashew-sensitized animals. More importantly, EPIT was able to reduce mast cell reactivity and to afford protection against anaphylactic symptoms following oral challenge, suggesting that EPIT may be a safe and efficacious approach to treat cashew allergy. During this work, we demonstrated that epicutaneous patches were able to deliver cashew allergen to skin dendritic cells (DC) using cashew protein extract conjugated to Fluoroprobe-647 (cashew-F647). We showed a significant increase of cashew-specific epidermal Langerhans cells, $\mathrm{cDC} 1$ and $\mathrm{cDC} 2$ in the local draining lymph nodes of mice that received patches containing cashew-F647 for 48 hours.

In the present work, we aimed to better characterize allergen capture at the skin level while evaluating the capacity of cashew allergen to modulate DC activation in sensitized or naïve animals. To that end, epicutaneous patches containing cashew-F647 were applied to cashew-sensitized or naïve mice for 6 hours (antibody titers measured before patch application are depicted on Figure $\mathbf{S 1}$ ). Then, skin was homogenized, and cells were analyzed by flow cytometry (Figure 1 ). Langerhans cells and cDC2 were the two main subsets of skin DC able to take up cashew allergen, in agreement with previously published data. ${ }^{4}$ Remarkably, a significant increase of cashew-positive Langerhans cells, $\mathrm{cDC} 2$ and $\mathrm{cDC} 1$ was observed in sensitized mice compared to naïve animals. Note that the difference was more pronounced for Langerhans cells, with a $47 \%$ increase of allergen-positive cells among this subset. To further address the activation status of these cashew-positive DC, the expression of CD86 and PD-L2 was measured in each skin DC subset (Figure 2 ). A significant decrease in CD86 expression was measured for cashew-positive Langerhans cells and cDC1 isolated in sensitized mice compared to naïve mice. Conversely, a significant increase of PD-L2 expression was observed in cashew-positive Langerhans cells and cDC2 isolated in sensitized animals.

Previous data demonstrated that PD-L2 expression by skin DCs, especially Langerhans cells and cDC2, is a key element leading to the generation of Tregs and to the acquisition of tolerance to topically administered allergen. ${ }^{4,5}$ Here, we demonstrated that the expression of PD-L2 and CD86 on skin DC following topical allergen application depends upon preexisting immunological status. Furthermore, these results support the fact that a strong Th2 context promotes the generation of tolerogenic skin DC characterized by a decrease in CD86 expression and an increase of PD-L2 expression, as previously shown by our group using OVA as a model allergen. ${ }^{6}$

Further investigations are warranted to decipher the mechanisms by which specific preexisting immunity impacts the capacity of skin DC to capture topically administered allergens and to modify their activation status. Our previous results suggest that preexisting IgG could efficiently promote allergen uptake (unpublished data), but the mechanisms leading to the modulation of CD86 and PD-L2 are still unknown.

Overall, these data provide additional insight into the mechanisms of action of EPIT for cashew allergy treatment. They suggest that the acquisition of tolerance to epicutaneous allergen would be more efficient in highly sensitized individuals and that the underlying mechanisms are embedded in the skin.

\section{References}

1. McWilliam V, Koplin J, Lodge C, Tang M, Dharmage S, Allen K. The Prevalence of Tree Nut Allergy: A Systematic Review. Curr Allergy Asthma Rep . 2015;15(54). doi:10.1007/s11882-015-0555-8

2. Clark AT, Anagnostou K, Ewan PW. Cashew nut causes more severe reactions than peanut: Case-matched comparison in 141 children.Allergy . 2007;62(8):913-916. doi:10.1111/j.1398-9995.2007.01447.x 
3. Pelletier B, Perrin A, Assoun N, et al. Epicutaneous immunotherapy protects cashew sensitized mice from anaphylaxis. Allergy . Published online 2020. doi:10.22541/au.159466842.25611016

4. Tordesillas L, Lozano-Ojalvo D, Dunkin D, et al. PDL2+ CD11b+ dermal dendritic cells capture topical antigen through hair follicles to prime LAP+ Tregs. Nat Commun . 2018;9(1). doi:10.1038/s41467-018-077167

5. Dioszeghy V, Mondoulet L, Laoubi L, et al. Antigen uptake by Langerhans cells is required for the induction of regulatory $\mathrm{T}$ cells and the acquisition of tolerance during epicutaneous immunotherapy in OVA-sensitized mice. Front Immunol . 2018;9(1951). doi:10.3389/fimmu.2018.01951

6. Laoubi L, Sampson HA, Mondoulet L, Nicolas J, Dioszeghy V, Vocanson M. Skin Dendritic Cells Progressively Subvert The Activation Of Pathogenic Type-2 Immunity Upon Epicutaneous Allergen Immunotherapy. $J$ Allergy Clin Immunol . 2019;143(2). doi:10.1016/j.jaci.2018.12.739

7. Hervé P-L, Dhelft V, Plaquet C, et al. Epidermal micro-perforation potentiates the efficacy of epicutaneous vaccination. J Control Release . 2019;298:12-26. doi:10.1016/j.jconrel.2019.02.004

\section{Legend to the figures}

Figure 1: Delivery of cashew allergens by epicutaneous patches leads to enhanced allergen capture by skin DCs in sensitized mice. Mice orally sensitized to cashew or naïve mice received a patch loaded with cashew-F647 (in green) or a patch containing excipient as a negative control (in white), for 6 hours. Skin samples were collected, and cells were isolated and labelled for FACS analysis. The percentage of F647-positive cells was measured among each skin DC subsets, $(\mathrm{n}=8$ per group). Data are median and interquartile ranges. $\mathrm{P}$ values were determined according to the Mann-Whitney test $\left(* *, \mathrm{p}<0.01\right.$; ${ }^{* * *}$, $\mathrm{p}<0.001)$.

Figure 2: Cashew-positive skin DC isolated in cashew-sensitized mice show an increased expression of PD-L2 and a concomitant decrease of CD86 expression. Mice were treated as described in Figure 1 and skin cells were analyzed by FACS. The expression of PD-L2 and CD86 was evaluated in F647-positive cells by measuring the median of fluorescence intensity of each marker. ( $\mathrm{n}=8$ per group). Data are median and interquartile ranges. $\mathrm{P}$ values were determined according to the Mann-Whitney test $\left(*, \mathrm{p}<0.05 ;{ }^{* *}, \mathrm{p}<0.01\right)$.

\section{Legend to supplementary figure}

Figure S1: Measurement of cashew-specific antibody titers in sensitized animals. Two groups of mice were orally sensitized to cashew (in green) while two groups were kept naïve as a negative control (in white). These two groups in each treatment regimen were intended to receive patches containing cashew-F647 or patches containing excipient. Blood samples were collected one week after the end of the sensitization, before patch application to isolate plasma. Cashew-specific antibody titers were measured from plasma by indirect ELISA (IgE) or direct ELISA (IgG1 and IgG2a). ( $=8$ per group). Data are median and interquartile ranges. The level of significance measured between sensitized and naïve groups is indicated above each histogram. $\mathrm{P}$ values were determined according to the Mann-Whitney test $\left(* *, \mathrm{p}<0.01\right.$; ${ }^{* * *}$, $\mathrm{p}<0.001)$.

\section{Supplementary methods}

\section{Animals and ethics.}

BALB/c mice were purchased from Charles River (Lyon, France) and housed under conventional conditions (DBV Technologies, Montrouge, France, agreement number \#A92-049-02). Experiments have been performed according to the European Community rules of animal care, and with permission of the French government (authorization \#7811).

\section{Extraction of cashew allergens for sensitization.}


Proteins were extracted from acetone-defatted cashew flour (Stallergenes Greer) by overnight stirring in PBS 1X, at $4^{\circ} \mathrm{C}$. The solution was centrifuged for $30 \mathrm{~min}$ at $3000 \mathrm{x}$ g to eliminate insoluble components. Supernatant was frozen at $-80^{\circ} \mathrm{C}$ and lyophilized (Lyofal). Nitrogen inertization was performed. Solutions for sensitization and oral challenge were obtained by adding the required volume of PBS $1 \mathrm{X}$ into vials. The final protein concentration was controlled using the Bradford assay.

\section{Sensitization of mice.}

Mice received $1 \mathrm{mg}$ of cashew protein extract supplemented with $10 \mu \mathrm{g}$ of Cholera Toxin (List Biological Laboratories) per intragastric route, at a rate of one intragastric administration per week for 6 consecutive weeks.

\section{Preparation of cashew patches and application to mice.}

Epicutaneous patches were loaded with $50 \mu \mathrm{g}$ of cashew protein extract conjugated to Fluoroprobe-647 (Interchim), prepared in phosphate buffer $0.1 \mathrm{M}$, or $50 \mu$ l of phosphate buffer $0.1 \mathrm{M}$ alone (excipient patches). Patches were dried at $30^{\circ} \mathrm{C}$ in a ventilated oven and stored at $4^{\circ} \mathrm{C}$. Before patch application, mice were anaesthetized with ketamine and xylazine (50 and $10 \mathrm{mg} / \mathrm{kg}$, respectively) and hair on the back was removed using electric clippers and depilatory cream (Reckitt Benckiser). Patches were applied the following day and secured using an Urgoderm ${ }^{\circledR}$ bandage (Urgo Laboratories). Patches were maintained for 6 hours based on optimal timepoints previously defined.

\section{Collection of plasma.}

Blood samples were collected by submandibular puncture into microtubes containing EDTA (Greiner BioOne) and centrifugated at $3000 \mathrm{x}$ g for 10 minutes to collect plasma. Plasma samples were stored at $-20^{\circ} \mathrm{C}$.

\section{Measurement of antibody titers.}

IgG1 and IgG2a titers were measured by direct ELISA: 96-well plates were coated overnight at $4^{\circ} \mathrm{C}$ with cashew protein extract $(5 \mu \mathrm{g} / \mathrm{mL}$, protein equivalent) or with anti-IgG1 or anti-IgG2a (5 or $1 \mu \mathrm{g} / \mathrm{mL}$, respectively) for binding of standards. Plates were washed in PBS 1X-Tween ${ }^{\circledR} 0.05 \%$ and blocked for 1 hour at room temperature (RT) with phosphate $0.1 \mathrm{M} \mathrm{pH} 7.4-\mathrm{NaCl} 0.15$ M-BSA $0.1 \%$-sodium azide $0.01 \%$ (EIA buffer). Plates were then incubated overnight at $4^{\circ} \mathrm{C}$ with mouse plasma (in duplicates) or serially diluted standards (Rabbit F(ab')2 anti-mouse IgG1 or Human anti-mouse IgG2a, Bio-Rad), and then 1 hour at $37^{\circ} \mathrm{C}$ with relevant secondary antibodies conjugated to alkaline phosphatase (AP) (Bio-Rad). Finally, plates were incubated for 30 minutes at RT with AP substrate (p-Nitrophenyl Phosphate, pNPP) and optical densities (O.D.) at $405 \mathrm{~nm}$ were recorded. Antibody concentrations were calculated with Boltzmann sigmoidal equation using O.D. obtained from standards (GraphPad Prism ${ }^{\circledR}$ ), and after subtracting O.D. obtained from non-specific points. IgE titers were measured by indirect ELISA to avoid competition with IgG1 binding: 96-well plates were coated with $2 \mu \mathrm{g} / \mathrm{mL}$ of rat anti-mouse IgE (Bio-Rad) and incubated overnight at $4^{\circ} \mathrm{C}$. Plates were washed in PBS $1 \mathrm{X}$-Tween ${ }^{\circledR} 0.05 \%$ and blocked 1 hour at RT with EIA buffer. Following washing, plates were incubated overnight at $4^{\circ} \mathrm{C}$ with mouse plasma (in duplicates) or serially diluted standard (mouse IgE, Bio-Rad), and then 1 hour at RT with biotinylated cashew proteins for plasma samples or biotin antimouse IgE (1:2000, Biolegend) for standard. Plates were finally incubated 1 hour at RT with AP-conjugated streptavidine (1:5000, Jackson Immuno Research Lab), and then 15 minutes at RT with pNPP. O.D. were recorded and antibody concentrations were measured as described above.

\section{Collection of skin samples for Flow Cytometric analysis (FACS).}

A skin sample corresponding to the patch application area was harvested using an 8-mm disposal biopsy punch (KAI medical) and transferred into $1 \mathrm{~mL}$ of Liberase TM (Roche) prepared in basic medium (RPMI + $\mathrm{PS}+55 \mu \mathrm{M}$ BME $+20 \mathrm{mM}$ HEPES), then incubated for 2 hours at $37^{\circ} \mathrm{C}$. The enzymatic reaction was then stopped with $75 \mu \mathrm{L}$ of EDTA $(100 \mathrm{mM})$. Cells were homogenized using the Medimachine tissue homogenizer (BD Bioscience) for 8 min.

Flow Cytometry analysis. 
Cell suspensions were incubated for $15 \mathrm{~min}$ at $4^{\circ} \mathrm{C}$ with Fc Block (BD Biosciences) and then stained for 30 min at $4^{\circ} \mathrm{C}$ with the following fluorochrome-conjugated antibodies: anti-XCR1-APC-Vio770 (clone: REA707, Miltenyi Biotec), anti-MHC-II-VioBlue (clone: REA813, Miltenyi Biotec), anti-CD11b-PerCP-Vio700 (clone: REA592, Miltenyi Biotec), anti-EpCAM-PE-Vio770 (clone: caa7-9G8, Miltenyi Biotec), anti-CD86-FITC (clone: PO3.3, Miltenyi Biotec) and anti-PD-L2-PE (clone: MIH37, Miltenyi Biotec). Cells were acquired on a MACSquant 16 flow cytometer (Miltenyi Biotec) and data were analyzed using FlowJo software using the gating strategy previously described. ${ }^{7}$ F647 fluorescence was measured using the R1 channel.

\section{Statistical data analysis.}

Data are presented as median with interquartile ranges. The non-parametric Mann-Whitney test was used to compare unpaired values (GraphPad Prism@). Values of $\mathrm{p}<0.05$ were considered significant. The level of significance is indicated with asterisks: ${ }^{*}, \mathrm{p}<0.05$; $^{* *}, \mathrm{p}<0.01{ }^{* * *}, \mathrm{p}<0.001$; ****, $\mathrm{p}<0.0001$ and n.s., non-significant.

\section{Hosted file}

Figures.pdf available at https://authorea.com/users/341526/articles/486711-capture-ofcashew-allergen-by-skin-dendritic-cells-is-enhanced-in-cashew-sensitized-mice-followingepicutaneous-delivery 